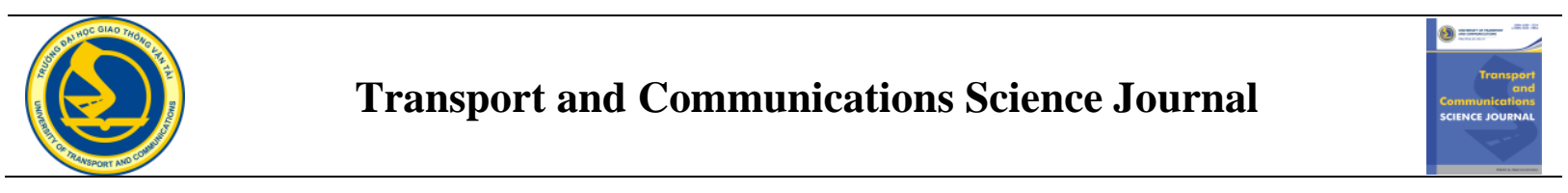

\title{
FUZZY-BASED QUANTIFICATION OF CONGESTION FOR TRAFFIC CONTROL
}

\section{Toan Trinh Dinh}

Department for Transportation Engineering, 422 Block A11, Thuy Loi University, 175 Tay Son, Dong Da, Hanoi, Vietnam

\author{
ARTICLE INFO \\ TYPE: Research Article \\ Received: 5/10/2020 \\ Revised: 30/10/2020 \\ Accepted: 6/11/2020 \\ Published online: 25/01/2021 \\ https://doi.org/10.47869/tcsj.72.1.1 \\ * Corresponding author \\ Email: Trinhdinhtoan@tlu.edu.vn; Tel: 0368420106
}

\begin{abstract}
In this work, a methodology for appraisal of congestion level for traffic control on expressways using fuzzy logic is presented. The congestion level indicates the severity of congestion and is estimated using speed and density, being the basic traffic parameters that describe state of a traffic stream. Formulation of the fuzzy rule base is made based on knowledge on traffic flow theory and engineering judgments. Field data on a segment of the Pan-Island Expressway of Singapore were used to estimate the congestion levels for three scenarios: single input variable (speed or density) and combined input variables (speed and density), represented by congestion level on a [0 1] scale. The results showed that there were big gaps between the congestion levels evaluated based specifically on speed and density alone (single state variable), and the congestion levels estimated from both variables lie in between. Given the uncertainty in traffic data collection and dynamic nature of traffic flow, this indicates that it may be inadequate to evaluate traffic congestion level using a single variable, and the use of both speed and density represent the state of a traffic stream more properly. The study results also show that the fuzzy logic approach provides flexible combination of state variables to obtain the congestion level and to describe gradual transition of traffic state, which is particularly important under the heavy congested conditions.
\end{abstract}

Keywords: Fuzzy Set; Fuzzy Rule; Congestion Level; Traffic State. 


\section{INTRODUCTION}

Quantification of congestion level is essential for congestion management. So far, efforts have been made to find ways to evaluate congestion level on roadway segments: For examples: [1] used density as a parameter to describe operational conditions for basic freeway segments; [2] evaluated the traffic congestion on urban highways based on speed, travel time, and demand/capacity ratio; [3] proposed several indicators to evaluate traffic congestion, including average travel speed, road saturation degree, loss of speed, and low-speed proportion.

In reviewing the previous works, it can be seen that a majority of studies focused on evaluation of traffic congestion for transport planning and traffic management under urban context, but little is known about research on quantifying congestion for traffic control on expressways. The planning and management applications primarily use the network performance measures to evaluate network congestion. There are practical constraints to use these measures for traffic control since they require time-consuming calculation through complicated procedures. Traffic control on expressways should use direct measurements of traffic variables to issue control actions in a real-time basis.

\subsection{Fuzzy Logic Applications in Traffic Engineering}

Fuzzy logic is a qualitative approach that is close to human observation, reasoning and decision-making. A fuzzy logic system (FLS) is a non-linear mapping of input to the output universe of discourse using fuzzy logic principles. The key motivations behind the use of fuzzy logic for quantification of congestion for traffic control include: (i) linguistic expressions are general and easy to be perceived, which is important in dealing with abstract nature of congestion [4]; (ii) its capability to combine several input quantities to produce a single output; and (iii) the transition from one fuzzy set to another is gradual, representing continuity in human perception.

There have been many studies that use fuzzy logic to evaluate traffic congestion: for example [3] used fuzzy logic to evaluate road traffic congestion level based on comprehensive parameters; [5] proposed a fuzzy inference approach to evaluate congestion level on arterial roadways using average speed and proportion of time traveling at very low speed; other research works on application of fuzzy logic approach for quantification of traffic congestion can be seen in [6] and [7]. However, most of the mentioned studies focused on specific applications, and little is known about evaluation of congestion level for traffic control on expressways.

This paper presents a methodology to quantify the congestion level on expressways using fuzzy logic for traffic control. For comparison, the congestion level is evaluated with three scenarios: single input variable (speed or density) and combined input variables (speed and density). The output (congestion level) is represented by congestion level (CL) on a [0 1] scale.

\subsection{Choice of Input Variables}

Speed is among the most common indicators of congestion since it reflects the mobility of traffic stream. Speed is relatively easy to be obtained through field surveys. The use of speed as an indicator of congestion is straightforward and intuitive. Many researchers have used speed to define congestion level such as $[8,9]$. Density is another primary measure for characterizing operational conditions on expressways. In [1] density is used as the only 
quantity to estimate the levels of service (LOSs) for basic segments on freeways.

In traffic flow theory, the general relationship been speed and density is typically described as linear relationship such as the Greenshields model. If speed and density were ideally linearly correlated, the use of a single measure would be surficial to represent the congestion condition. However, previous studies indicate that speed may be constant while the flow rate increases up to $1,300 \mathrm{pc} / \mathrm{h} / \mathrm{lane}$, but decreases significantly just before the flow rate approaches the capacity [1]. Considering the complex and dynamic nature of traffic, it may be difficult to comprehensively assess traffic congestion conditions by a single evaluation indicator [10]. The use of both of speed and density may be necessary to represent the operational conditions of expressway traffic: density reflects freedom to maneuver, and speed is a major concern of drivers as related to service quality. They are both quantitative measures that characterize operational conditions of a traffic stream on expressway. Speed is a direct measurement, while density can be provided by detection cameras installed at a vantage point or is easily derived from occupancy.

\section{DEVELOPMENT OF FUZZY RULES FOR CONGESTION LEVEL}

\subsection{Membership Functions}

In a multiple input - single output (MISO) fuzzy structure, the inputs are called state variables, and the output is called control variable. To express the state variables in a high resolution, we category each state variable into 5 predicates (Fig. 1):

$$
\begin{aligned}
V & =\{\text { Very_low, Low,Medium, High,Very_high }\} \\
K & =\{\text { Very_low, Low,Medium, High, Very_high }\}
\end{aligned}
$$

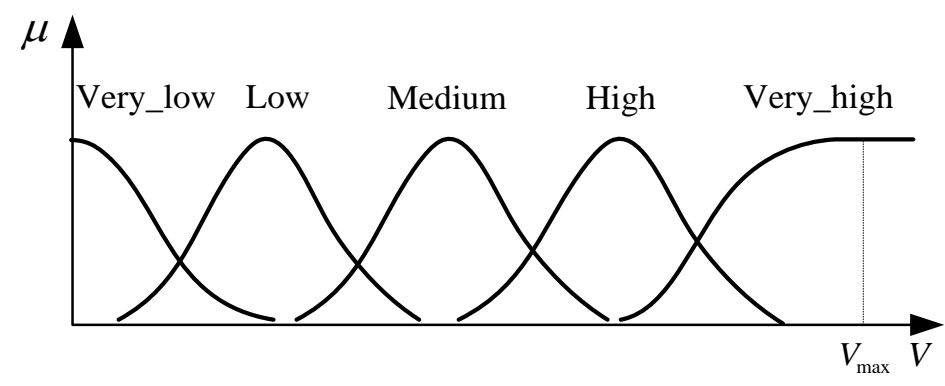

a) Speed

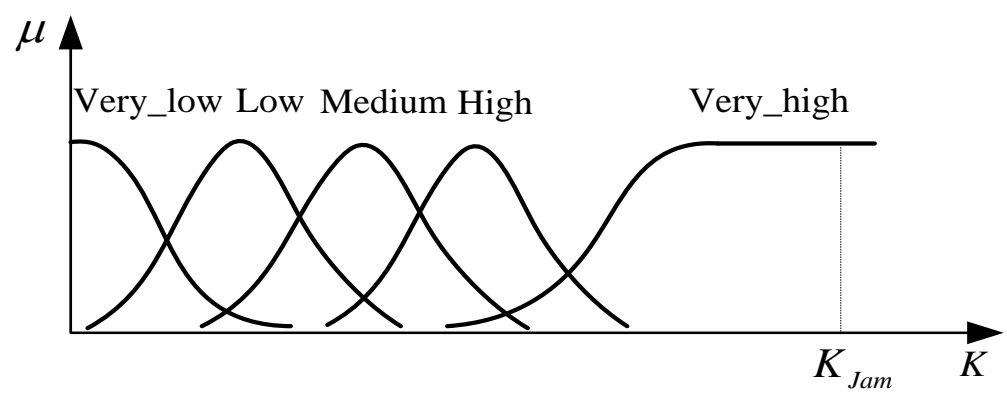

b) Density

Figure 1. Fuzzy partition of state variables. 
Transport and Communications Science Journal, Vol. 72, Issue 1 (01/2021), 1-8

and the control variable is partitioned into 5 fuzzy sets (Fig. 2):

$$
C L=\{\text { FreeFlow, Ligh, Moderate, Heavy,VeryHeavy }\}
$$

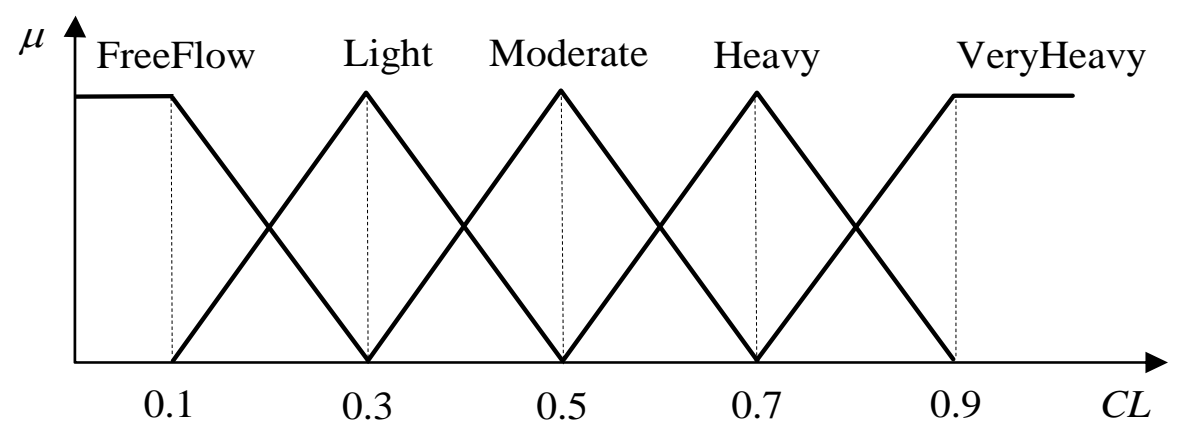

Figure 2. Fuzzy partition of control variable (CongestionLevel).

where FreeFlow is associated with LOS A and partly to LOS B; Light congestion corresponds to LOS C and partly to LOSs B and D, with speed reducing, flow increasing and the freedom to maneuver within the traffic stream is noticeably limited; Moderate congestion describes operation that approaches the road capacity (LOS E) and partly to LOS D, where speed deceases significantly, density increases quickly with increasing flows, and maneuverability within the traffic stream is limited; Heavy congestion describes breakdowns in vehicular flow, which can be considered as approaching the LOS F at which point queues may form with potential propagation upstream. Finally, VeryHeavy represents an extreme breakdown of flow of very low traffic dynamics. It is strictly associated with LOS F.

\subsection{Formation of Rules}

Rules for the congestion level are characterized by two predicates in the antecedent, connected with an AND operator, and one predicate in the consequent. The general expression of rules is of the form:

$$
\text { If speed is } V_{(x)} \text { AND density is } K_{(x)} \text { then congestion level is } C L_{(x)}
$$

Table 1. Rule decision matrix for congestion level.

(FF: Free flow, L: Light congestion, M: Moderate congestion, H: Heavy congestion, VH: Very

\begin{tabular}{|c|c|c|c|c|c|}
\hline \multicolumn{6}{|c|}{ Density } \\
\hline Relation & VeryLow & Low & Medium & High & VeryHigh \\
\hline VeryLow & --- & --- & $\mathrm{H}$ & VH & VH \\
\hline Low & --- & M & M & $\mathrm{H}$ & VH \\
\hline Medium & $\mathrm{L}$ & $\mathrm{L}$ & M & $\mathrm{H}$ & $\mathrm{H}$ \\
\hline High & $\mathrm{FF}$ & $\mathrm{L}$ & M & M & --- \\
\hline VeryHigh & $\mathrm{FF}$ & $\mathrm{FF}$ & $\mathrm{L}$ & --- & --- \\
\hline
\end{tabular}
heavy congestion)

The formation of the rules for the congestion level is conducted in the same way as the preliminary rule set: in the left-hand side, the two predicates are connected with the AND 
operator, thus the membership values are calculated using the MIN operation.

An example of rule can be seen as follow:

If $\mathrm{V}$ is "VeryLow" AND $\mathrm{K}$ is "VeryHigh" then CL is "VeryHeavy"

The collection of the rules includes 19 rules as summarized in Table 1 that is known as rule decision matrix for congestion level.

Although the maximum combinations of the state variables constitute $5 * 5=25$ rules, some combinations are not meaningful, and are removed from the model, including: "VeryHigh" speed - "VeryHigh" density, "VeryHigh" speed - "High" density, "High" speed "VeryHigh" density. We note that the relationship "VeryLow" speed - "VeryLow" density, "Low" speed - "VeryLow" density, and "VeryLow" speed - "Low" density may happen under specific circumstances such as incident or roadwork during the nighttime. However, from the database investigation, there were no such situations during the data collection period. Since in a uninterrupted-flow facility, the cause of congestion is internal [1], we remove these combinations because they are unlikely to occur.

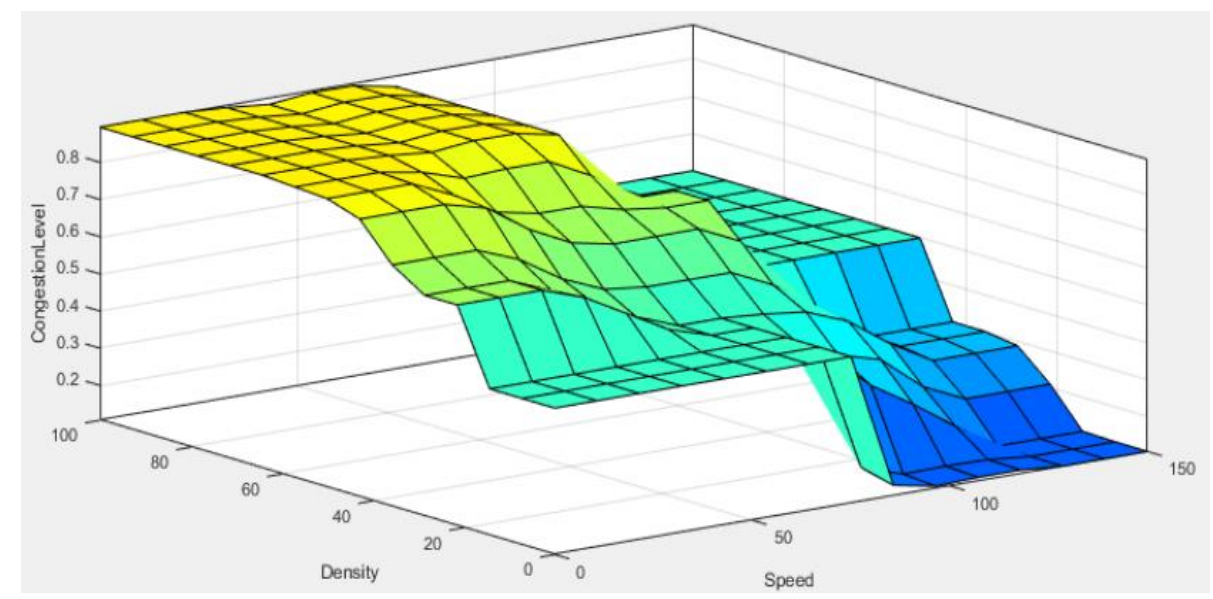

Figure 3. Surface Viewer of congestion level.

Fig. 3 examines the output space of the fuzzy logic system at a $5 \times 5$ resolutions of fuzzy inputs (Speed and Density) that produce a 5 resolution of output (CongestionLevel).

\section{EVALUATION OF CONGESTION LEVELS USING ACTUAL DATA}

This section demonstrates the evaluation of congestion level using data obtained on a segment of Pan-Island Expressway of Singapore. Since the data are point measurements, they do not permit direct calculation of speed and density. Speeds used in this analysis were space mean speeds $\left(\bar{U}_{s}\right)$ that are estimated from the measured time mean speeds $\left(\bar{U}_{t}\right)$ using Eq. (5) for time mean speeds of less than $70 \mathrm{~km} / \mathrm{h}[1]$.

$$
\bar{U}_{s} \approx 1.026 \times \bar{U}_{t}-1.89
$$

For a time mean speed that is greater than $70 \mathrm{~km} / \mathrm{h}$, space mean speed is approximated as $2 \%$ less than the time mean speed with reference from traffic engineering literature [1]. 
Transport and Communications Science Journal, Vol. 72, Issue 1 (01/2021), 1-8

Direct measurement of density in the field is difficult since this requires a vantage point for photographing over a significant segment of roads. In this study, density is calculated as the ratio of flow rate $\mathrm{V}$ (veh./lane/h) to the space mean speed $\bar{U}_{s}(\mathrm{~km} / \mathrm{h})$.

$$
K=\frac{V}{\bar{U} s}
$$

To explore further findings from previous studies that using the hybrid index is more reliable and practical than results using a single index, the congestion levels evaluated using both speed and density $\mathrm{CL}\left(\mathrm{v}^{*} \mathrm{k}\right)$ are compared with the congestion levels evaluated using a single quantity, that is either speed (CL(v)) or density $(\mathrm{CL}(\mathrm{k}))$. Three methods use the same sets of membership functions proposed in Fig. 1.

The CL( $\left.\mathrm{v}^{*} \mathrm{k}\right)$ used the rule base as described in Section 2.2. Using speed as the only input variable, the input-output mapping of $\mathrm{CL}(\mathrm{v})$ is implemented by the following simple set of rules:

- If speed is VeryLow then congestion level is VeryHeavy

- If speed is Low then congestion level is Heavy

- If speed is Medium then congestion level is Moderate

- If speed is High then congestion level is Light

- If speed is VeryHigh then congestion level is FreeFlow.

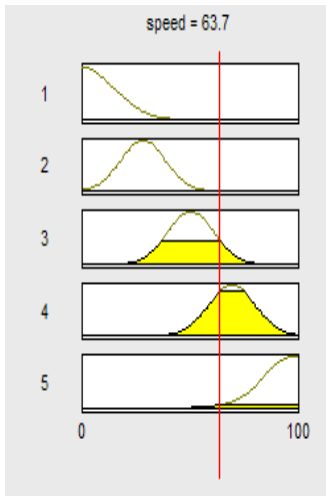

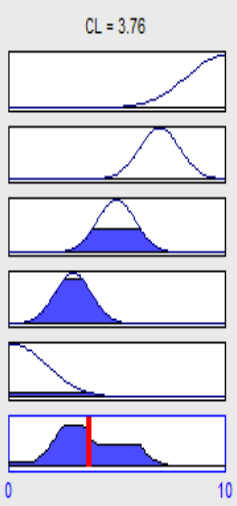

a) $\mathrm{CL}(\mathrm{v})$

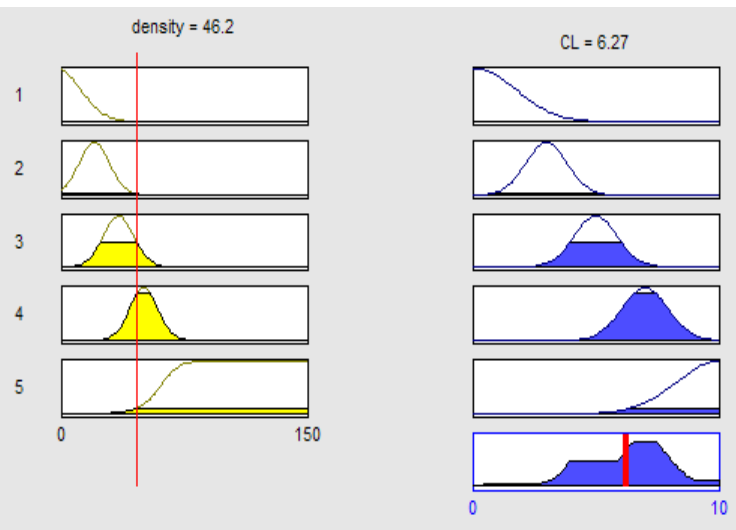

b) $\mathrm{CL}(\mathrm{k})$

Figure 4. The rule interface for the state variables.

Similarly, using density as the only input variable, the input-output mapping of CL(k) are obtained using the simple set of rules:

- If density is VeryLow then congestion level is FreeFlow

- If density is Low then congestion level is Light

- If density is Medium then congestion level is Moderate

- If density is High then congestion level is High

- If density is VeryHigh then congestion level is VeryHeavy. 
The membership functions of CL(v) and CL(k) are illustrated in Figure 44.

To illustrate what have been discussed in the introduction regarding the number of state variables, the congestion levels estimated using both speed and density $\mathrm{CL}\left(\mathrm{v}^{*} \mathrm{k}\right)$ are compared with those using a single quantity (Fig. 5), either speed (CL(v)) or density (CL(k)). Fig. 5 shows that there are large differences between the congestion levels evaluated by CL(v) and $\mathrm{CL}(\mathrm{k})$ under free-flow and light congested conditions. In principle, if CL(v) and CL(k) were good indicators of congestion level, they would be sufficiently close since they used the same set of data. This proves that it would be inadequate to represent the congestion level using a single variable of speed or density. The congestion level using both speed and density will provide better representation of traffic conditions.

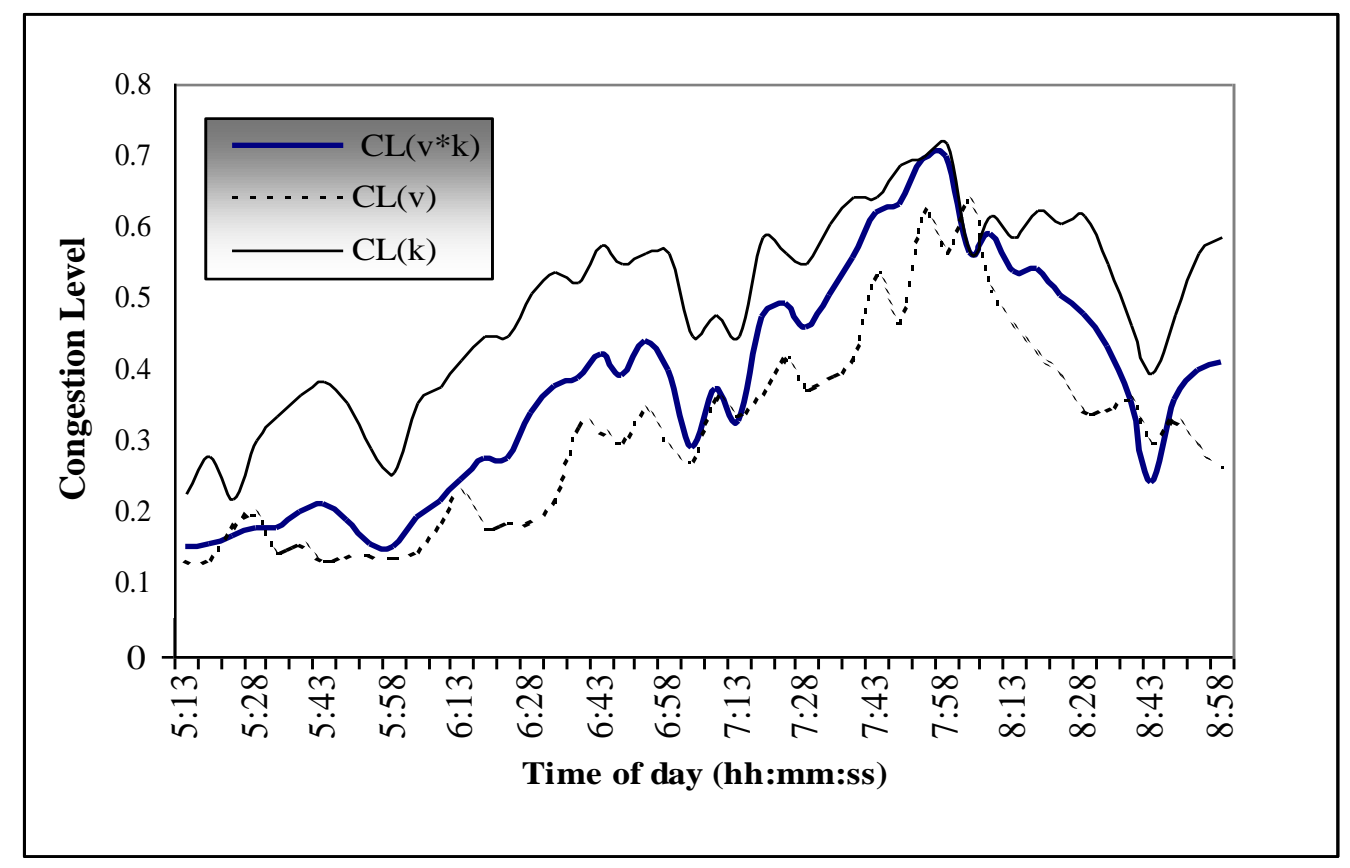

Figure 5. Evaluation of congestion levels by different methods.

\section{CONCLUSION}

The control of traffic on a road network in general, on expressways in particular, requires evaluation of traffic conditions, specifically the congestion intensity under critical conditions. The congestion intensity (congestion level) is a vague concept in nature, and is dependent on a number of parameters, hence the evaluation of congestion level requires a decision-support system that is able to combine several inputs to evaluate the output (congestion level) and effectively convey the output in a manner that is easily to be perceived by the traffic control officers.

This paper presents a methodology for quantification of congestion level for traffic control on expressways following the fuzzy logic approach (FLS). The congestion level indicates the severity of congestion and was evaluated by using speed and density, being the primary traffic variables: speed is a direct measurement, while density can be provided by detection cameras installed at a vantage point, or is easily derived from occupancy. The output (congestion level) is represented on a [0 1] scale that can be further categorized into classes, upon which appropriate control actions are issued. 
The FLS used field data on a segment of the Pan-Island Expressway of Singapore to estimate the congestion levels for three scenarios: single input variable (speed or density) and combined input variables (speed and density). The results showed that there are remarkable differences between the congestion levels evaluated with either speed or density alone, while the congestion level profile constructed using both variables lies in between. This indicates that it would be inadequate to represent the congestion level using a single variable of speed or density. Considering the complexity and dynamic nature of traffic, it is necessary to quantity traffic congestion using both variables.

The study results also show that the FLS provides flexible combination of input variables to obtain the congestion level and to describe gradual transition of traffic state, which is particularly important under heavy congested conditions.

\section{ACKNOWLEDGEMENTS}

To demonstrate the fuzzy-based methodology, this paper used data obtained on a segment of Pan-Island Expressway of Singapore. The author would like to gratefully acknowledge the Land Transport Authority of Singapore for its provision of data used in this study.

\section{REFERENCES}

[1]. Highway Capacity Manual, Sixth Edition: A Guide for Multimodal Mobility Analysis, Washington, D.C. (2020).

[2]. A. M. Rao, K. R. Rao, Measuring Urban Traffic Congestion - a Review, International Journal for Traffic and Transport Engineering, 2 (2012) 286-305. https://doi.org/10.7708/ijtte.2012.2(4).01

[3]. W-X. Wang, R-J. Guo, J. Yu, Research on Road Traffic Congestion level Based on Comprehensive Parameters: Taking Dalian City as an Example, Advances in Mechanical Engineering, 10 (2018) 1-8. https://doi.org/10.1177/1687814018781482

[4]. L. A. Zadeh, Fuzzy Logic, Neural Networks, and Soft Computing. Communication of the ACM, 37 (1994) 77. https://doi.org/10.1145/175247.175255

[5]. K. Hamad, S. Kikuchi, Developing a Measure of Traffic Congestion - Fuzzy Inference Approach, Transportation Research Record: Journal of the Transportation Research Board, 1802 (2002) 77-85. https://doi.org/10.3141/1802-10

[6]. M. Arabani, S. Pourzeynali, Fuzzy Logic Methodology to Evaluate the Service Level of Freeways Basic Segments, Iranian Journal of Science \& Technology Transaction B: Engineering, 29 (2005) 281288. https://citeseerx.ist.psu.edu/viewdoc/download?doi=10.1.1.515.4924\&rep=rep1\&type=pdf

[7]. M. D. Munajat, D. Widyantoro, R. Munir, A New Method for Analyzing Congestion Levels Based on Road Density and Vehicle Speed, Journal of Theoretical and Applied Information Technology, 95 (2017) 6454-6471. http://www.jatit.org/volumes/Vol95No23/10Vol95No23.pdf

[8]. Bertini, R. Lawrence, You Are the Traffic Jam: An Examination of Congestion Measures, Presented at 85th Annual Meeting of the Transportation Research Board, Washington, D.C., (2006) 1419.

[9]. D. C. Xiao, S. Yun, An Improved Non-Linear Fuzzy Comprehensive Method for Assessing Urban Freeway Network Traffic Congestion Level, Open Journal of Transportation Technologies, 9 (2020) 68-78. https://doi.org/10.12677/OJTT.2020.92009

[10]. F. He et al., A Traffic Congestion Assessment Method for Urban Road Networks Based on Speed Performance Index, Procedia Engineering, $137 \quad$ (2016) 425-433. https://doi.org/10.1016/j.proeng.2016.01.277 